\title{
On a model of an unconstrained hyperfluid
}

\author{
Yuri N. Obukhov* \\ Institute for Theoretical Physics, University of Cologne, D-50923 Köln, Germany
}

\begin{abstract}
A hyperfluid is a classical continuous medium carrying hypermomentum. We modify the earlier developed variational approach to a hyperfluid in such a way that the Frenkel type constraints imposed on the hypermomentum current are eliminated. The resulting self-consistent model is different from the Weyssenhoff type one. The essential point is a conservation of the hypermomentum current such that the final metrical and canonical energy-momentum forms coincide.

PACS no.: 04.40.+c; 04.50.+h
\end{abstract}

Typeset using REVTEX

*On leave from: Department of Theoretical Physics, Moscow State University, 117234 Moscow, Russia 


\section{INTRODUCTION}

Recently [1] the variational model of a hyperfluid has been developed. This is a classical continuous medium, elements of which possess both polarizability and elasticity properties. Such a matter might be a non-quantum source of the metric-affine gravity [2] within the framework of the gauge theory for the general affine space-time symmetry group. The hyperfluid model in [1] is constructed as a natural generalization of the phenomenological Weyssenhoff spin fluid [3], the variational theory of which was elaborated in [四] (in the Lagrange approach) and in [5] (in the Euler approach). Although the Weyssenhoff model, in our opinion, provides a reasonable description for a spin fluid, it is well known that there exist different models of continuous media with spin [6 8]. Likewise, the properties of matter with hypermomentum are not sufficiently well known at present. Thus a study of possible different hyperfluid models appears to be a necessary step in the development of this subject.

In the present paper we discuss a possibility of removing the standard Frenkel type constraints usually imposed on the hypermomentum current.

\section{PRELIMINARIES: MATTER CURRENTS IN METRIC-AFFINE GRAVITY}

Our basic notations and conventions are that of the review [2], except for the metric signature which assumed here to be $(+,-,-,-)$. The field equations of the metric-affine gravity theory are derived from the general Lagrangian four-form $L=V+L_{\text {mat }}$, with $V=$ $V\left(g_{\alpha \beta}, \vartheta^{\alpha}, Q_{\alpha \beta}, T^{\alpha}, R_{\alpha}^{\beta}\right)$ and $L_{\mathrm{mat}}=L_{\mathrm{mat}}\left(g_{\alpha \beta}, \vartheta^{\alpha}, \Gamma_{\alpha}{ }^{\beta}, \Psi, D \Psi\right)$ as the pure gravitational and matter Lagrangians, respectively. Here the metric 0 -form $g_{\alpha \beta}$, coframe 1 -form $\vartheta^{\alpha}$, and affine connection 1 -form $\Gamma_{\alpha}^{\beta}$ are the gravitational potentials, the respective field strengths are nonmetricity 1-form $Q_{\alpha \beta}:=-D g_{\alpha \beta}$ and the 2-forms of torsion $T^{\alpha}$ and curvature $R_{\alpha}{ }^{\beta}$ [2]. The general field equations read

$$
2 \frac{\delta V}{\delta g_{\alpha \beta}}=-\sigma^{\alpha \beta}, \quad \frac{\delta V}{\delta \vartheta^{\alpha}}=-\Sigma_{\alpha}, \quad \frac{\delta V}{\delta \Gamma_{\alpha}^{\beta}}=-\Delta_{\beta}^{\alpha},
$$


where the matter currents are defined as the variational derivatives

$$
\sigma^{\alpha \beta}:=2 \frac{\delta L_{\mathrm{mat}}}{\delta g_{\alpha \beta}}, \quad \Sigma_{\alpha}:=\frac{\delta L_{\mathrm{mat}}}{\delta \vartheta^{\alpha}}, \quad \Delta^{\alpha}{ }_{\beta}:=\frac{\delta L_{\mathrm{mat}}}{\delta \Gamma_{\alpha}{ }^{\beta}} .
$$

It worthwhile to mention that the standard Einstein-Hilbert Lagrangian $V=-\frac{1}{2} R_{\alpha}^{\beta} \wedge *\left(\vartheta^{\alpha} \wedge\right.$ $\left.\vartheta_{\beta}\right)$ (considered in the recent paper [9], e.g.) is a bad choice for metric-affine gravity. Due to an auxilliary (so-called projective, see [2]) invariance, this Lagrangian is only compatible with the dilaton-free sources for which $\Delta=\Delta^{\alpha}{ }_{\alpha}=0$, and the Weyl 1-form $Q=g^{\alpha \beta} Q_{\alpha \beta}$ is not determined by the gravitational field equations (2.1).

The Weyssenhoff type hyperfluid [1] is described by the following currents: the metric stress-energy 4-form

$$
\sigma^{\alpha \beta}=\eta\left((\varepsilon+p) u^{\alpha} u^{\beta}-p g^{\alpha \beta}\right)+2 u^{\gamma} u^{(\alpha} D \Delta_{\gamma}^{\beta)}
$$

the canonical energy-momentum 3-form

$$
\Sigma_{\alpha}=u P_{\alpha}-p\left(\eta_{\alpha}-u u_{\alpha}\right)
$$

and the hypermomentum 3-form

$$
\Delta_{\beta}^{\alpha}=u J_{\beta}^{\alpha},
$$

where $\varepsilon$ is the energy density, $p$ the pressure, $u$ the flow 3 -form, $J_{\beta}^{\alpha}$ the hypermomentum density, and $P_{\alpha}$ the four-momentum

$$
P_{\alpha}=*\left(\varepsilon u \wedge \vartheta_{\alpha}-2 u^{\beta} g_{\gamma[\alpha} D \Delta_{\beta]}^{\gamma}\right)
$$

As usually, $\eta^{\alpha}=* \vartheta^{\alpha}$ and $\eta$ is the volume 4-form. Components of 4-velocity are defined by $u=u^{\alpha} \eta_{\alpha}$. Hypermomentum includes as irreducible parts the spin density $S_{\alpha \beta}=J_{[\alpha \beta]}$ and the dilaton charge $J=J^{\alpha}{ }_{\alpha}$.

Hypermomentum density satisfies the constraint,

$$
J_{\beta}^{\alpha} u^{\beta}=J_{\beta}^{\alpha} u_{\alpha}=0
$$

This is a natural generalization of the Frenkel condition $S_{\alpha \beta} u^{\beta}=0$. 
Recently [10] it has been suggested that the generalised Frenkel condition could be weakened and, in particular, reduced just to the ordinary Frenkel constraint imposed on the spin part of the hypermomentum density. Below we study the possibility of constructing the completely unconstrained hyperfluid model.

\section{VARIATIONAL PRINCIPLE FOR THE UNCONSTRAINED HYPERFLUID}

We start from the Lagrangian 4-form for the hyperfluid, cf. [1],

$$
\begin{gathered}
L_{\mathrm{mat}}=\varepsilon\left(\rho, s, \mu_{B}^{A}\right) \eta-\frac{1}{2} \rho \mu_{B}^{A} b_{\alpha}^{B} u \wedge D b_{A}^{\alpha}- \\
-\rho u \wedge d \lambda_{1}+\lambda_{2} u \wedge d X+\lambda_{3} u \wedge d s+\lambda_{0}(* u \wedge u-\eta)+\lambda_{B}^{A}\left(b^{B} \wedge b_{A}-\eta \delta_{A}^{B}\right) .
\end{gathered}
$$

Here the first two terms describe the internal energy density $\varepsilon$ and the kinetic energy density, respectively. As usually, we assume that the internal energy of hyperfluid depends on the particle density $\rho$, the specific entropy $s$ and the specific hypermomentum density $\mu_{B}^{A}$. It seems worthwhile to notice at this point that the indices $A, B, \ldots$, which run from 1 to 3 , are merely labels and they have no any geometrical meaning. The material frames $b^{A}$ (which is a 1-form with an expansion $b^{A}=b_{\alpha}^{A} \vartheta^{\alpha}$ ) and $b_{A}$ (which is a 3-form with analogous expansion $b_{A}=b_{A}^{\alpha} \eta_{\alpha}$ ) have nothing to do with the reference frames which an observer may introduce and change (i.e. rotate and deform) at his own choice. The material frames together with the specific hypermomentum are a sort of internal variables which describe the polarizability and elasticity properties of the fluid elements. Material frames are rigidly attached to elements of the continuum and their evolution is determined by the equations of motion of the fluid. From the geometrical point of view, $\mu_{B}^{A}$ is a scalar and $\varepsilon$ is explicitly a generally coordinate invariant quantity. The energy density cannot be a function of the hypermomentum density tensor, as is incorrectly assumed in [9], since this is incompatible with the general coordinate invariance of the fluid action. 
The remaining terms in (3.1) describe constraints. The first three express the conservation particle number, constancy of entropy, and identity of elements along the flow lines:

$$
\begin{gathered}
d(\rho u)=0, \\
u \wedge d X=0, \\
u \wedge d s=0 .
\end{gathered}
$$

The last two terms in (3.1) tells that the fluid velocity is timelike and normalized,

$$
* u \wedge u=\eta
$$

and that material frame variables are dual in the sense

$$
b^{B} \wedge b_{A}=\delta_{A}^{B} \eta
$$

Here is the crucial modification of the hyperfluid model [1]. Recall that the material triad is rigid in the spin fluid model (which can only rotate), while in accordance with the affine gauge approach we assumed that in a hyperfluid the material frame $b_{A}^{\alpha}$ is elastic and can deform arbitrarily during the motion of the medium. However, in the Weyssenhoff type model [1] the generalized material tetrad $\left(u, b_{A}\right)$ is still constrained in that the material triad is assumed to be orthogonal to the velocity, $* u \wedge b_{A}=0$. Now, developing the suggestion of [10], we remove the latter orthogonality condition. Hereafter such a medium is called unconstrained hyperfluid.

The derivation of the Euler-Lagrange equations is analogous to [1]. As before, the independent variables are: the metric-affine gravitational potentials $g_{\alpha \beta}, \vartheta^{\alpha}, \Gamma_{\beta}^{\alpha}$, the material variables $\Psi=\left\{b_{A}, b^{B}, \rho, \mu_{B}^{A}, s, X\right\}$, and the Lagrange multiplier 0-forms $\lambda_{0}, \lambda_{1}, \lambda_{2}, \lambda_{3}, \lambda_{B}^{A}$.

Varying the action (3.1) with respect to the Lagrange multipliers, one finds (3.2)-(3.6). Variations of $s, X, \rho, \mu_{B}^{A}$ and $u, b^{B}, b_{A}$ yield the equations, respectively,

$$
\eta\left(\frac{\partial \varepsilon}{\partial s}\right)+d\left(\lambda_{3} u\right)=0, \quad d\left(\lambda_{2} u\right)=0
$$




$$
\begin{gathered}
\eta\left(\frac{\partial \varepsilon}{\partial \rho}\right)-\frac{1}{2} \mu_{B}^{A} b_{\alpha}^{B} u \wedge D b_{A}^{\alpha}-u \wedge d \lambda_{1}=0 \\
\eta\left(\frac{\partial \varepsilon}{\partial \mu_{B}^{A}}\right)=\frac{1}{2} \rho b_{\alpha}^{B} u \wedge D b_{A}^{\alpha} \\
-\frac{1}{2} \rho \mu_{B}^{A} b_{\alpha}^{B} D b_{A}^{\alpha}-\rho d \lambda_{1}+\lambda_{2} d X+\lambda_{3} d s-2 \lambda_{0} * u=0 \\
\frac{1}{2} \rho \mu_{B}^{A} *\left(u \wedge D b_{A}^{\alpha}\right) \eta_{\alpha}+\lambda_{B}^{A} b_{A}=0 \\
\frac{1}{2} * D\left(\rho \mu_{B}^{A} b_{\alpha}^{B} u\right) \text { varthet } a^{\alpha}+\lambda_{B}^{A} b^{B}=0 .
\end{gathered}
$$

These equations determine the Lagrange multipliers. While $\lambda_{1}, \lambda_{2}, \lambda_{3}$ satisfy differential evolution equations, one can explicitly solve (3.8)-(3.11) with respect to the other Lagrange multipliers,

$$
\begin{gathered}
2 \lambda_{0}=\rho\left(\frac{\partial \varepsilon}{\partial \rho}\right), \\
\lambda_{B}^{A}=\frac{1}{2} \rho \mu_{B}^{C} *\left(u \wedge b_{C}^{\alpha} D b_{\alpha}^{A}\right) .
\end{gathered}
$$

Substituting (3.14) back into (3.11) and (3.12), we get the system of equations of motion of the specific hypermomentum density and the material frames:

$$
\begin{gathered}
u \wedge\left(d \mu_{B}^{A}+\mu_{C}^{A} b_{B}^{\alpha} D b_{\alpha}^{C}-\mu_{B}^{C} b_{C}^{\alpha} D b_{\alpha}^{A}\right)=0, \\
\left(\delta_{\alpha}^{\beta}-b_{\alpha}^{C} b_{C}^{\beta}\right) \mu^{A}{ }_{B} u \wedge D b_{\beta}^{B}=0, \\
\left(\delta_{\alpha}^{\beta}-b_{\alpha}^{C} b_{C}^{\beta}\right) \mu^{A}{ }_{B} u \wedge D b_{A}^{\alpha}=0 .
\end{gathered}
$$

While (3.15) coincides with the analogous equation of motion for the Weyssenhoff type hyperfluid [1], equations (3.16)-(3.17) are new. One can rewrite (3.15)-(3.17) in terms of the tensor variables, demonstrating that these equations express the conservation of hypermomentum current. Indeed, contracting (3.15) with $b_{A}^{\alpha} b_{\beta}^{B}$ and using (3.16)-(3.17), we find 


$$
u \wedge D\left(\mu_{B}^{A} b_{A}^{\alpha} b_{\beta}^{B}\right)=0
$$

The matter currents of the unconstrained hyperfluid are derived by direct calculation of variational derivatives (2.2). Denoting as usually [1],

$$
J_{\beta}^{\alpha}=\frac{1}{2} \rho \mu_{B}^{A} b_{A}^{\alpha} b_{\beta}^{B},
$$

and introducing the pressure in a standard way,

$$
p:=\rho\left(\frac{\partial \varepsilon}{\partial \rho}\right)-\varepsilon
$$

one obtains (using (3.15)-(3.18)):

$$
\begin{gathered}
\sigma^{\alpha \beta}=\eta\left((\varepsilon+p) u^{\alpha} u^{\beta}-p g^{\alpha \beta}\right), \\
\Sigma_{\alpha}=\varepsilon u u_{\alpha}-p\left(\eta_{\alpha}-u u_{\alpha}\right), \\
\Delta_{\beta}^{\alpha}=u J_{\beta}^{\alpha} .
\end{gathered}
$$

The most essential difference of the resulting unconstrained model from the Weyssenhoff type hyperfluid [1] is the complete decoupling of hypermomentum from both energy 3forms (3.21) and (3.22). This is compatible with the Noether identities [2] though, as the equation (3.18) (multiply by $\rho$ and use (3.4) ) actually describes the conservation of the hypermomentum current,

$$
D \Delta_{\beta}^{\alpha}=0
$$

Dynamics of all irreducible parts of hypermomentum is contained in (3.24), and it is indeed unconstrained since neither the Frenkel type conditions (2.7), nor any other are imposed on the hypermomentum density.

The complete decoupling of $\Delta^{\alpha}{ }_{\beta}$ from the energy currents of course does not mean that unconstrained hypermomentum is not affecting the gravitational field: it still enters as the source in the third metric-affine gravity field equation (2.1). 
At the first sight, it may seem surprising that such a simple modification of the original hyperfluid model, as the elimination of orthogonality condition of the material triad and velocity, may yield the above described essential change of the equations of motion of hypermomentum and reduce the energy-momentum 3-form to that of the ideal structureless fluid. However, everything becomes clear when one notices that the Lagrangian (3.1) of the unconstrained hyperfluid possesses an extra gauge symmetry besides the usual coordinate and local gravitational frame $G L(4, R)$ invariances. Namely, (3.1) is invariant under the simultaneous transformations of the material frames and the affine connection components,

$$
b_{A}^{\alpha} \longrightarrow L_{\beta}^{\alpha} b_{A}^{\beta}, \quad b_{\alpha}^{B} \longrightarrow b_{\beta}^{B} L_{\alpha}^{-1 \beta}, \quad \Gamma_{\alpha}^{\beta} \longrightarrow L_{\sigma}^{\beta}\left(\Gamma_{\rho}^{\sigma}+\delta_{\rho}^{\sigma} d\right) L_{\alpha}^{-1 \rho},
$$

where $L_{\beta}^{\alpha} \in G L(4, R)$. Notice that this is different from the affine gauge gravity transformation, since the gravitational frame $\vartheta^{\alpha}$ and metric $g_{\alpha \beta}$ are not transformed. The total Lagrangian $L=V+L_{\text {mat }}$ certainly does not possess the symmetry (3.25). Applying to the Lagrangian (3.1) the Noether machinery [2], one can derive a conservation law corresponding to (3.25). This is exactly (3.24).

\section{DISCUSSION AND CONCLUSION}

In the Weyssenhoff type hyperfluid [1] the generalized Frenkel condition (2.7) plays an important role. It indeed restricts the possible motions of the medium, as well as the very structure of the hypermomentum current. In particular, (2.7) rules out the case of purely dilatonic Weyssenhoff matter. Using the standard decomposition [2] of hypermomentum

into its irreducible parts, $J_{\beta}^{\alpha}=g^{\alpha}{ }_{\beta}+\frac{1}{4} J \delta_{\beta}^{\alpha}+S_{\beta}^{\alpha}$, one finds in view of (2.7) that the dilaton charge is expressed in terms of the proper hypermomentum (shear) according to $J=-4 \nexists_{\alpha \beta} u^{\alpha} u^{\beta}$. Thus vanishing shear yields also $J=0$. Unlike this, the unconstrained hyperfluid may be of the purely dilatonic type. In this case our model gives a description of a physical source for the generalized Einstein-Weyl gravity theory considered recently in [11. 
In [10] an attempt was made to construct a sort of intermediate model in which the constraint (2.7) reduces to the original Frenkel condition, $F_{\alpha}=S_{\alpha \beta} u^{\beta}=0$. Technically this can be achieved if one adds to (3.1) the term $\xi^{\alpha} F_{\alpha}$ with a Lagrange multiplier (4-form) $\xi^{\alpha}$. Such a term breaks the symmetry (3.25). However the resulting equations of motion for the fluid and the gravitational currents (2.2) look unusual and their physical interpretation is unclear. Contrary to the expectations of the authors of [10 (who even failed to solve the highly nontrivial system of constraint equations with respect to $\xi^{\alpha}$ ), at the end one does not find a Weyssenhoff type dynamics for the spin part of the hypermomentum.

It should be noted that the described above unconstrained hyperfluid is not a subcase of the general Weyssenhoff hyperfluid model. They are close though, in the sense that the two theories may admit the same particular solutions for the gravitational and matter field equations. The next decisive step will be a comparison of different ideal hyperfluid models [1.9,10] with the real physical media the elements of which display polarizability and/or elasticity properties.

\section{Acknowledgements}

I would like to thank Friedrich W. Hehl for stimulating discussions and useful comments. This research was supported by the Deutsche Forschungsgemeinschaft under the project He-528/17-1. 


\section{REFERENCES}

$1 \quad$ Yu.N. Obukhov and R. Tresguerres, Phys. Lett. A184 (1993) 17.

2 F.W. Hehl, J.D. McCrea, E.W. Mielke and Yu. Ne'eman, Phys. Rept. 258 (1995) 1.

3 J. Weyssenhoff and A. Raabe, Acta Phys. Polon. 9 (1947) 7.

$4 \quad$ W. Kopczyński, Phys. Rev. D34 (1986) 352.

$5 \quad$ Yu.N. Obukhov and V.A. Korotky, Class. Quantum Grav. 4 (1987) 1633.

$6 \quad$ J.R. Ray and L.L. Smalley, Phys. Rev. Lett. 49 (1982) 1059.

$7 \quad$ R. De Ritis, M. Lavorgna, G. Platania and C. Stornaiolo, Phys. Rev. D28 (1983) 713.

$8 \quad$ H.P. de Oliveira, Gen. Relat. Grav. 25 (1993) 473.

$9 \quad$ L.L. Smalley and J.P. Krisch, J. Math. Phys. 36 (1995) 778.

10 O.A. Babourova and B.N. Frolov, The variational theory of perfect fluid with intrinsic hypermomentum in space-time with nonmetricity, Preprint Moscow Pedagogical State University, LANL e-archive gr-qc/9509013 (1995) 7p.

11 R.W. Tucker and C. Wang, Class. Quantum Grav. 12 (1995) 2587. 